\title{
Evaluation of Water Needs Index Case Studies
}

\author{
$\underline{\text { K.S. Alexander }}^{\mathrm{a}}$, M. Moglia ${ }^{\mathrm{b}}$, G. Tjandraatmadja ${ }^{\mathrm{b}}$, M. Nguyen ${ }^{\mathrm{b}}$, S. Larson $^{\mathrm{c}}$, N.H. Trung ${ }^{\mathrm{d}}$ and R.A. Barkey $^{\mathrm{e}}$ \\ ${ }^{a}$ CSIRO Ecosystem Sciences, Canberra, Australian Capital Territory \& Charles Sturt University, School of \\ Environmental Sciences, Institute of Land, Water and Society, Albury, NSW, ${ }^{b}$ Urban and Industrial Water \\ Research Program, CSIRO Land and Water, Victoria, ${ }^{c}$ CSIRO Ecosystem Sciences, Townsville, QLD. , ${ }^{C}$ Can Tho \\ University, Can Tho, Vietnam, ${ }^{e}$ Hasanuddin University,Makassar, Indonesia. (Email: kim.alexander@,csiro.au)
}

\begin{abstract}
Adoption of a systems perspective by water planners responsible for infrastructure and supply can provide considerable benefits, even though analysis of water systems can be a very daunting task. Furthermore, actors in the system are often subject to individual biases, cognitive limitations, and often have limited timelines and resources. In addition, a paucity of pertinent information and spatial and temporal data limitations are problematic to water resource decision makers who are subject to bounded rationality. To overcome some limitations inherent in water resource management decisions, the authors have used exploratory techniques combining actor engagement with data collection and analysis using the Water Needs Index (WNI). The WNI methodology is a structured approach to assess multiple dimensions of water needs of particular spatial environments. The WNI has been previously applied at different scales (national, regional, catchment and urban) and has been found to be of most use at catchment and urban scales. The WNI methodology applies a mix of qualitative and quantitative approaches in case study settings. The process involves a review of several data sources (quantitative records and grey literature) for selection and compilation to calculate a WNI for a range of spatial locations. Qualitative research methods, such as workshops, have been used to investigate subjective opinions and incorporate local contextual knowledge. Workshops with key actors from urban water management case study areas have been found to be useful in facilitating dialogue and establishing dimensions, identifying useful and reliable data sources and adding insights and confirmation of appropriateness of data selection.
\end{abstract}

This research reports on several case studies using the WNI methodology, in the Philippines, Viet Nam and Indonesia. The WNI methodology has been used to inform development of preliminary information embedded in Integrated Urban Water Management (IUWM) approaches and Integrated Water Resource Management (IWRM) which take a more holistic systems' perspective when planning for urban water management. The WNI index is a relatively straightforward multi-criteria assessment (using weights) forming a two-level hierarchy of dimensions and data sources, with systems analysis. The key task is the definition of dimensions, and the choice of data sources, as either observations or proxies of the dimensions. Selection of dimensions and subsequent ranking of water needs become an integral part of the development of the IUWM and IWRM. These approaches provide a means to manage water resources more sustainably by accounting for natural flows, water quality, water extraction, and balancing environmental and community needs. By taking a systems perspective and using index methodology, the views of stakeholders can be considered in the decision-making process and inform planning for more efficient use of resources. The research shows that the WNI is useful in informing urban water management planning and for provision of information essential to more holistic water resource outcomes.

Activities have been designed to achieve a number of objectives for each case study city. Activities were designed to collectively frame the enquiry by taking a systems' perspective and using an observational framework by identifying data sources and integrating diverse knowledge sources. Engaging with a mix of stakeholders with often conflicting goals required a process for dialogue between actors and between institutions and enable conflict resolution to take place if required. The primary research outcome was development of a preliminary systems analysis. This research explores whether workshop interactions and data gathering activities achieved each project's stated objectives. This paper reports on the success or otherwise in meeting project objectives by introducing an evaluation process, based on the Protocol of Canberra.

Keywords: Case study research, Water Needs Index, research evaluation, research methods 


\section{INTRODUCTION}

Although there is a vast amount of information on the current status, future demand and impact of climate change on water supply and services, data accessibility and usefulness for decision making is contentious. There are many difficulties in deciding on the important factors to consider in the management of water resources. Adoption of a systems perspective by water planners responsible for infrastructure and supply management can be beneficial, even though analysis of water systems can be a very daunting task. Participatory modelling, an approach combining participatory activities with modelling techniques, can be used to assist collective decisionmaking in natural resource management (Jones et al. 2009). Gathering and integrating local experts' viewpoints can assist modelling and the development of a collective vision for the management of common resources. One participatory modelling approach is the Water Needs Index (WNI), which draws on the Climate Vulnerability Index by Sullivan and Meigh (2005). The WNI undertakes a systems analysis using a weighted multi-criteria assessment with a hierarchy of dimensions and data sources. The key task is the definition of dimensions, and the choice of data sources, as either observations or proxies of the dimensions. While the mathematical computation is straightforward, constructing the index and accessing suitable data is difficult and complex, with considerable subjective judgment required in the choice of dimensions. Reliability and accuracy of the data sources is important. Alexander et al. (2008) discusses the inherent difficulties of observation that occur when using proxies. The WNI index typically incorporates physical, economic and social factors, using a deliberative process with stakeholders to determine the water needs of a range of locations (Alexander et al., 2009, Moglia et al., 2008). This process is a post-normal scientific approach, that is useful when all factors are not necessarily knowable and where accessing all relevant information is too time consuming or unclear and affected by high levels of uncertainty (Funtowicz and Ravetz 1993). The WNI methodology has been used to inform development of preliminary information embedded in Integrated Urban Water Management (IUWM) approaches and Integrated Water Resource Management (IWRM) which take a more holistic systems' perspective when planning for urban water management, as used in research by Maheepala et al. (2010). These approaches provide a means to manage water resources more sustainably by accounting for natural flows, water quality, water extraction, and balancing environmental and community needs. The WNI approach can achieve several functions in bolstering the effectiveness and accuracy of the IUWM framework, as identified by Jones et al. (2009) by; increasing the legitimacy (normative function), increasing knowledge (substantive function) and building collaborative relationships across traditional institutional boundaries (instrumental function). To evaluate the functional aptitude of the WNI approach used at several case study sites, the Protocol of Canberra (Jones et al., 2009) has been undertaken.

\section{USING THE PROTOCOL OF CANBERRA EVALUATION FRAMEWORK}

The Protocol of Canberra designed by Jones et al. (2009), has been used to evaluate projects by identifying the; (i) the context and objectives of each project, (ii) the process (the methods and tools used) and (iii) underpinning theoretical assumptions. Curnan et al. (1998) believe that underpinning most projects is an explicit or implicit theory describing the workings of the project, commonly known as the 'espoused theory' (Argyris, 1999). Espoused theory, or what should happen in a project, differs from 'theory-in use' which is what really happens. The Protocol of Canberra evaluation framework seeks to capture the espoused theory by working with a project team to identify the sequence of methods used and their anticipated effects. Patton (1992) claims that the analysis can compare the situated ideals (espoused theory) with real priorities (theory-in-use) to understand the reasons for and implications of discrepancies. Curnan et al. (1998) suggest that by identifying specific methods (and their corresponding tools) associated with explicit effects, more can be understood about the most influential factors and the project's impact. This paper describes the influential factors that have been elicited using concepts from the Protocol of Canberra (Jones et al., 2009), where we have undertaken a formative evaluation by capturing initial experiences of project teams and the logic of their research design. This is an iterative process which will be used to modify research design and future activities. The evaluation has included a review of project personnel opinions, reports and communications that have outlined theoretical assumptions and objectives. The WNI method has been applied in three cases: Iloilo City, Philippines; Makassar City, South Sulawesi, Indonesia and Can Tho, Viet Nam, with varying degrees of success.

\section{CASE CONTEXT}

\subsection{Iloilo City, Philippines}

The port city of Iloilo, Panay Island, Philippines, has a population of 300,000, and is the commercial and cultural hub of the island (Alexander et al. 2009). The city sources water from the Tigum Aganan Watershed 
and residents have been subject to poor water quality, lack of sanitation, increasing siltation, decreasing water availability, groundwater contamination, the threat of saltwater intrusion into the aquifer, and catastrophic floods and droughts. The productivity of the river has been compromised by headwater surges, pollution from mining activities, riverbank erosion, reduced fish habitat and the impacts of urban migration. Natural hazards such as landslides and erosion are exacerbated by heavy rains in the uplands, threatening the lives and livelihoods of village communities. Over-exploitation of resources, social injustices, governance issues and rural poverty are problematic. Watershed stakeholders established a multi-sector management body of over 40 stakeholders in 2000, in response to a history of watershed management issues and the cessation of flow in the river (Salas et al. 2009). A research workshop was held with stakeholders to assess management of watershed resources and inform decisions about land use, natural resource management and infrastructure investment. The question guiding this research was: Can disputed environmental management issues be resolved through a mediated scientific-stakeholder process incorporating conceptual systems modelling and evidence-based decision support tools? The water needs of the city were assessed using the WNI methodology which used six dimensions in the assessment as suggested by Sullivan and Meigh (2005); Resources, Capacity, Use, Access, Environment and Vulnerability; with each populated by a number of sub-indicators.

\subsection{Can Tho City, Vietnam}

Can Tho City is one Vietnam's major cities, located in the Mekong River Delta with a population of about 1.2 million people, with $65 \%$ of those living in rural and sub-urban areas (Moglia et al., 2011). Can Tho City is aspiring to be the political, economic, and social-cultural centre of Mekong Delta region. During the last two decades economic growth and urbanisation of the region have increased substantially with the development of more residential, commercial, and industrial areas. The rapid growth rate of Can Tho City and peri-urban regions has put great pressure on environmental conditions with many pollution problems, in particular, water pollution, wastewater, solid waste, and environmentally related diseases (World Bank, 2003). As a consequence of the rapid urbanisation, Can Tho is struggling to meet basic requirements for sustainable urban development and has many challenges in designing climate-adapted built environments. The question guiding this research was: How to support decision making for water and wastewater infrastructure investment in Can Tho that reflects and responds to the anticipated impacts of climate change, population and urbanisation? The Water Needs Index (WNI) was modified through discussions with stakeholders and used to explore urban water issues according to six dimensions; Aquatic Ecosystems, Flooding, Groundwater, Infrastructure Systems, Water and sanitation access, and Water Quality, with some interest expressed in including governance issues. A workshop was conducted with key technical and managerial staffs from a range of stakeholder organisations, including relevant government departments, the local water utility, and local institutes.

\subsection{Makassar City, Sulawesi Island, Indonesia}

Makassar is the capital city of the South Sulawesi Province and is highly urbanised with an estimated population of 1.2 million in 2006, set to increase significantly in the future. An important issue is adaptation to sea level rise with the threat of inundation and salt water intrusion potentially impacting important groundwater resources and could cause degradation of sub-surface assets, limiting the adequacy of water supplies into the future. Makassar is already struggling to meet the demand for clean water supply and in 2009 the municipal water company supply network covered $74 \%$ of the total population in the city (Alexander et al., 2011). Given the plans for the future growth of the city, a feasibility study has been developed for construction of a wastewater treatment plant and for a solid waste and landfill management facility (Muryanto, 2011). The overarching research question was: How to support decision making for water and wastewater infrastructure investment in Makassar that reflects and responds to the anticipated impacts of climate change as well as other key drivers for growth such as population and urbanisation. The WNI was considered the basis for two assessments, for the water supply catchments and urban issues of Makassar City, based on the six indices used in the Iloilo City example, with sub-indices modified to suit after consultation with stakeholders. The workshops were conducted with the assistance of Hasanuddin University, and other key government and utility stakeholders. In developing the WNI, discussions centred on sub-indices and their relative importance and of weighting the dimensions. Sub-indicators for each dimension and weightings were determined by self-selected groups and were discussed in plenary. Overall assessments of the water needs of the region are yet to be completed, though will incorporate opinions, knowledge, and consensual agreements that will be validated in future discussions.

\section{METHODS}

Project teams, goals and workshop designs differed for all case studies, but all projects were situated in rapidly developing cities of South East Asia, dealing with increasing populations, resource degradation, polluted 
waterways and inadequate water and sanitation supplies. Projected climate change has significant implications for water resource planning at each site. Case details and research outcomes are described in detail in reports, and journal and conference papers by the authors (Alexander et al., 2009, Alexander et al., 2008, Alexander et al., 2010, Alexander et al., 2011, Moglia et al., 2008, Moglia et al., 2010). The authors assume that the WNI provides an example of participatory modelling, and hence the focus of this paper is on the evaluation of the three projects following process suggestions by Jones et al. (2009). Several key steps involved in constructing the indexes involving deliberative processes are important to evaluate including; (i) meeting objectives, (ii) interacting with stakeholders, (iii) implementing the workshop and (iv) index development. Evaluations of the key steps were compiled by the authors to compare and contrast the application and usefulness of the WNI approach.

\section{RESULTS}

\subsection{Index development, rankings and usefulness}

Sullivan and Meigh (2005) designed an index to highlight water poverty and vulnerability to climate change using available data. In this research concepts have been furthered using a deliberative process to engage with local stakeholders and embed knowledge into the process of index development and their calculations. In practice at the local scale, theoretical index methodology is supported by engagement which leads to social learning and co-development of participatory modelling. Indexes resulting from these case studies have been designed to rank the water needs of locations and identify hotspots of concern to alert local stakeholders to problems and issues within their precincts of control. For Iloilo and Makassar Cities the ranking was of prime importance to inform IUWM framework assessments and to engage with local stakeholders to share knowledge and ensure the facilitation of linkages between organisations responsible for supplying water to burgeoning cities. In the Iloilo City study the main objective was providing a framework to enhance institutional relationships and interactions as a conflict resolution process. In Makassar and Can Tho Cities, the primary aims were to develop a common frame of reference, identify data sources and generating collaborative relationships. In Can Tho City, provision of contextual information and deliberative methods were more important than ranking water needs.

\subsection{Interacting with stakeholders}

Through evaluation, four main key stakeholders' attributes were found to be important factors in determining the ability to co-develop the projects; (i) project initiation, (ii) inclusion of key stakeholders, (iii) presence of project champion and (iv) institutional support for project. For example, in Makassar City, there was strong institutional support from central government for the project with the local, pro-active university the key stakeholder and contributor. Dedicated project personnel determined and engaged stakeholders, initiated preceding meetings and assisted in design and development of the process. In both Makassar and Can Tho City workshops and fieldtrips were organised over several days, for greater interaction and collegiate integration. In Can Tho City, prior discussions with Can Tho University, key stakeholders and personnel from other international aid projects, determined the list of key stakeholders. Organisations were invited to the workshop rather than individual invitations. Preceding the workshop, there was a formal ceremony of endorsement by government representatives, adding legitimacy. The local university provided strong facilitative support throughout the projects in Makassar and Can Tho Cities. .However, Iloilo City differed, as the project conception was initiated over the internet by a local champion representing a politically active organisation of watershed management at loggerheads with government catchment managers. Despite little institutional support, the organisational skills in bringing the stakeholders together were exceptional, with targeted personnel in attendance and opportunities for interactions over several days.

\subsection{Implementing the workshops}

The workshop implementation processes used in the three cases was compared, including issues such as: time schedules, facilitation, language and ability to capture information as the workshop proceeded. In Iloilo City, five day workshops and a field trip was well organised with over 40 key personnel in attendance. Several social events ensured that relationships were progressively built between stakeholders and research staff. Facilitation and presentations were conducted in English language with stakeholders working in groups to present written and verbal presentations, with general note-taking and observations by research colleagues. Accompanying the field trip to the headwaters of the watershed were 3 guards with semi-automatic rifles, indicating unresolved conflicts underpinned watershed management issues. In Makassar City, previous workshops had been used to 
familiarise all project staff with the main issues and introduce the key stakeholders. Workshops were scheduled over several days including a field trip to the main dam in the catchment, with social events designed to facilitate conversations and linkages. Some project staff members were bilingual (English and Bahasa Indonesia) and served to interpret proceedings, presentations and describe comments. A local facilitator was also enlisted for assistance. Project staff, if not bilingual, had more difficulty understanding the flow of knowledge in circumstances where proceedings were not predominantly in English language. University staff further assisted communications with data compilation and all gained valuable insights and learnings from the presentations by local water stakeholders. Note taking, recordings and translation occurred through trained local collaborators and project team members that spoke/read Bahasa. All original notes were checked so culturally specific meanings were not lost in translation. In Can Tho City, the process involved a one day workshop for an introduction of concepts by the primary researcher, providing opportunities for structured and unstructured, formal and at times informal discussions. During the workshop, participants added context and information about the dimensions and had an opportunity to establish and define index dimensions in constructive discussions. Workshop proceedings relied heavily on break-out groups facilitated by instructed local collaborators. Some presentations and group discussion were conducted in English language, though most of the workshop was presented in Vietnamese language. Note taking and translation occurred through trained local collaborators and a bilingual project team member who was able to interpret, read original notes to check translations. The workshop was followed up with formal meetings at various organisations to follow up on workshop outcomes.

\subsection{Index evaluation}

Evaluation of key issues in the development of the index was about participant definitions, stakeholder rankings, modelling software issues and dissemination of results. In Iloilo City, there were pre-workshop presentations and discussions of index and sub-indices prior to the workshop activities over several days and at sites in order to include most stakeholders. Index calculations were undertaken during workshops and maps were generated to provide presentations of rankings of municipalities using a simplified version for index computation and modelling. The final rankings included qualitative data from deliberative processes conducted during workshops, with reports and other communications finalising the project. The process was successful in terms of establishing a shared understanding of main issues and areas of concern, but the findings were not reviewed by decision makers. Neither the local water resource managers nor the funding agency who specified the participatory modelling tool acted on information gained through field testing, so the impact in terms of aiding decision processes may be small. In contrast, academic communications have been well received and the research organisation has supported ongoing development of WNI methods for use in further projects described herein. In Makassar City, participants defined the index dimensions through preliminary and iterative discussions and by building consensus without generating computing rankings or geographical representations. Two distinct indices were designed, firstly a catchment scale index which contrasted three catchments providing water for Makassar City and surrounds and secondly an index to rank the major issues that affected the urban supply of water and sanitation. Occasional deliberative processes were undertaken, particularly in communications with the local collaborating staff who continued to identify useful data for analysis, before and after the workshops. Compilation remains incomplete and when data have been established and used to calculate the WNI, another iterative process of deliberations are planned to confirm initial weightings. A future workshop will be undertaken to confirm and discuss the WNI rankings for catchments and for issues impacting supply of quality water to Makassar City. Engagement with local government staff involved in water supply in Can Tho

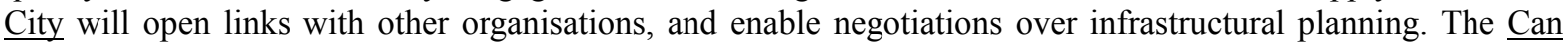
Tho City workshop focussed on concepts and in establishing the index and sub-components. Calculations were completed after the workshops and reported in English and Vietnamese languages. In Makassar and Can Tho City, qualitative information, data and recommendations will be used to complete an IUWM assessment and to finalise reports in English language and Bahasa. In both cities, training workshops for disseminated modelling skills are also planned to further build capacity in local universities.

\section{DISCUSSION}

In Iloilo City, the WNI approach was effective in opening dialogue and improving understanding of the functioning of the watershed in different municipalities, exploring the accessibility of quality water and discussing problems arising from natural hazards, particularly flooding events, in maintaining supplies of water. These discussions over index development with weighting of sub-components were critical in leveraging the full capacity of the WNI for overall assessments of the water needs of the region under discussion. Finalised assessments have embedded shared information, opinions, knowledge, and consensual agreements that are 
useful in validating results and gaining a more informed ranking of water needs. Research conducted at the watershed scale was well received by participants, which was articulated in evaluative feedback. A letter of confirmation of successful engagement was received as testament of stakeholders' appreciation of participatory nature of the research.

In Can Tho City, the workshop provided for constructive and useful discussions, fostering ways of thinking about the integrated nature of urban water issues. This is seen as a first step in improving the integrative and adaptive capacity of Can Tho water managers. From a project perspective, the workshop has achieved several important tasks including; (i) providing a qualitative description of the Can Tho water system, (ii) identifying key data and information sets and (iii) as an avenue providing active engagement with stakeholders and enabling critical dialogue to support discussions on future scenarios, as well as planning and designing alternative options for improved integrated urban water management. Stakeholders have been enthusiastic in collecting information identified in the workshop. Researchers also commented that cross-departmental and inter-disciplinary interaction was useful for enabling new ways of dealing with rapid urbanisation and climate change; many noting that the impacts were greater than most participants had expected. This raises the question as to whether a permanent community of practice for integrated discussions on urban water systems may be useful for ongoing capacity building. Workshop management by country partners created a greater sense of bi-lateral collaboration and increased capacity and organisational skills at the Can Tho University. This workshop therefore has been the first and critical step of the project, to be followed by data collection to further understand the current situation and future development opportunities. Understanding of the wider implications and consequences of climate change and urbanisation for Can Tho will set direction for subsequent activities to assess climate change impacts on water resources and water services. The WNI can be used to support other research methods such as systems analyses and development of future scenarios, to inform a more coordinated approach towards planning and safeguarding water supplies. Geographically referenced issues identified as components of the dimensions have been nominated as water risk hot spots, to be further researched in the development of alternative water service options. This indicates that the formation of an index using a workshop setting informed development of future research activities as per the research design.

It is difficult to judge the full impact of the formulation of the WNI in Makassar City as participants are not yet privy to the final assessment or recommendations for implementation. The workshop assisted in the development of a common understanding of problem/context among participants and fostered dialogue among parties. In Makassar City, communication more frequently occurs between government agencies and the university, but rarely occurs between government departments and NGOs. Descriptions of organisational linkages reveal that adaptive governance can be influenced through networks of communication and by complex systems of agencies, often with limited but specific responsibilities, so increasing linkages would be a project impact. The WNI seemed to be adding a sense of purpose for information sharing rather than only developing a common understanding of the context. During the workshop country partners were open and generously shared information and knowledge of issues with researchers from Australia and the local university (Hasanuddin University), all benefited from knowledge sharing. The workshop created an opportunity for an introduction of researchers' activities and further networking with country, provincial and local agencies. Having the management of the workshop conducted by country partners created a greater sense of bi-lateral international collaboration and fostered collaboration between agencies. A sense of collaboration was felt strongly by various actors as many were involved earlier at project planning phases. For example, discussions with the local university considerably influenced project and workshop planning, through actions of several project champions. An important outcome is an exchange in information and training for selected local university colleagues as well as more generalised capacity building from learnings of the project and the implementation process.

\section{CONCLUSION}

An initial evaluation and review of projects incorporating a WNI has been undertaken using the Protocol of Canberra. The WNI framework has been flexibly adapted to inform urban water resource management in three study sites, with varying success. The context, process and espoused theoretical assumptions (explicit and implicit) have been described. The 'theory-in-use' has been described more in terms of what really happened, with identification of the most influential factors and possible project impacts. While the evaluation has allowed for comparisons and insights, future evaluations will need a more structured and formalised approach. The benefits from combining participatory procedures with modelling techniques has been the sharing of knowledge and information between local experts and with researchers through a simple, understandable and flexible approach to gathering data, opinions and providing a means to explore contentious topics. Elicited information 
can then be used to assist collective decision-making processes and in the establishment of a collective vision for the future management of resources. Important insights into the impact of languages became apparent, as was the importance of liaison with local universities for improved project implementation and capacity building opportunities. Of upmost importance is that complex urban water resource systems require facilitation of networking opportunities, linkages and communication channels into agencies with responsibilities for ensuring quality water supplies. This appears to be an important role of the WNI framework, over and above the nominal ranking purpose of the modelling tool.

\section{ACKNOWLEDGMENTS}

Appreciation is expressed for significant contributions from CSIRO-AUSAID Alliance project team members, Dr Dewi Kirono, research partners from the Tigum Aganan Watershed, Dragon Institute, Can Tho University, Hasanuddin University, particularly Dr Jessica Salas, Profs Amran, Selitung, Yudono, Dharmawan, Kaimuddin, and colleagues, workshop contributors and R. Djalanti, from Macquarie University.

\section{REFERENCES}

Alexander, K.S., Miller, C., Jovanovic, T. \& Moglia, M. (2009). Tigum-Aganan Watershed Management Project, Part 2: Developing a Water Needs Index. In: PROJECT, C. A. A. (ed.) Climate Adaptation National Research Flagship Report. Canberra, Australia: CSIRO.

Alexander, K.S., Moglia, M. \& Austin, J. (2008). Regional and country scale water resource assessment. Informing investments in future water supply in the Asia Pacific region - a decision support tool. In: ALLIANCE, C.-A. (ed.) CSIRO Water for a Healthy Country National Research Flagship Report. Canberra, Australia: CSIRO.

Alexander, K.S., Moglia, M. \& Miller, C. (2010) Water Needs Assessment: learning to deal with scale, subjectivity and high stakes. Journal of Hydrology, 388(3-4), 251-257.

Alexander, K.S., Tjandraatmadja, G., Neumann, L., Kirono, D., Larson, S., Djalante, R., Barkley, R.A., Ahmad, A., Yudono, A., Dharmawan, A., Kaimuddin, K., Seliting, M. \& Primiantoro, E.T. (2011). Climate Adaptation through Sustainable Urban Development in Makassar, Indonesia. Water Needs Index. In: PROJECT, C. A. A. (ed.). Canberra, Australiqa: Climate Adaptation National Research Flagship.

Argyris, C. (1999) On organizational learning, Oxford,UK, Wiley-Blackwell.

Curnan, S., LaCava, L., Sharpstee, D., Lelle, M. \& Reece, M. (1998) W.K Kellogg Foundation evaluation handbook [Online]. Available: http://www.wkkf.org/pubs/Tools/Evaluation/Pub770.pdf [Accessed $12 / 06 / 2011]$.

Funtowicz, S.O. \& Ravetz, J.R. (1993) Science for the post-normal age. Futures 25(739-755.

Jones, N.A., Perez, P., Measham, T.G., Kelly, G.J., d’Aquino, P., Daniell, K.A., Dray, A. \& Ferrand, N. (2009) Evaluating Participatory Modeling: Developing a Framework for Cross-Case Analysis. Environmental Management, 44(6), 1180-1195.

Maheepala, S., Blackmore, J., Diaper, C., Moglia, M., Sharma, A. \& Kenway, S. (2010) Integrated Urban Water Management Planning Manual, Denver, CO, Water Research Foundation.

Moglia, M., Alexander, K.S., Cook, S., Sullivan, C., Lane, B. \& Lipkin, F. (2008). Regional and Country Scale Water Resource Assessment; Informing Investments in Future Water Supply in the Asia Pacific Region - a Decision Support Tool Water for a Healthy Country National Research Flagship Report Series. Melbourne, Australia: CSIRO.

Moglia, m., Cook, S., Nguyen, M., Trung, N.H., Paddon, M., Carrard, N., Lipkin, F. \& Maharg, S. (2010) water Risk Index Workshop in Can THo, Vietnam. Water for a Healthy Country National Research Flagship Report Series. Melbourne, Australia: CSIRO.

Muryanto, I. (2011). Metropolitan Sanitation Management and Health Project Makassar City presentation Pelayanan Air Bersih Dan Sanitasi Kota.

Salas, J., Miller, C., Alexander, K.S. \& Jovanovic, T.A. (Year) Towards democratic governance for a contested watershed: a Philippine case study. In: 12th International Riversymposium, 21-24 September 2009 2009 Brisbane, Australia. Proceedings of the 12th International Riversymposium.

Sullivan, C.A. (2002) Calculating a Water Poverty Index. World Development, 30(7), 1195-1210.

Sullivan, C.A. \& Meigh, J.R. (2005) Targeting attention on local vulnerabilities using an integrated index approach: the example of the climate vulnerability index. Wat. Sci. Tech., 51(5), 69-78.

World Bank (2003). Environmental Impact Assessment Can Tho City Sub-Project, Vietnam Urban Upgrading Project, E817, Volume 2. Can Tho City, Vietnam: Soil and Water Ltd, Finland in association with Duongthanh Water and Environment Ltd., . 